\title{
Viabilidade da pneumonectomia direita em cães: uma avaliação paramétrica, hemogasométrica e radiográfica ${ }^{1}$
}

\author{
Edson A. Simões ${ }^{2 *}$, Fabio B. Jatene ${ }^{3}$, Wanderley M. Bernardo ${ }^{3}$, Angelo J. Stopiglia ${ }^{4}$, \\ Rosangela Monteiro ${ }^{3}$, Denise T. Fantoni ${ }^{4}$, Cristina D. Abduch ${ }^{5}$, Karina V.B. Yasbek ${ }^{2}$, \\ Eduardo T. Irino ${ }^{2}$, Silvana M. Unruh ${ }^{4}$, Márcia Kahvegian² e André L.H. Soares ${ }^{2}$
}

\begin{abstract}
Simões E.A., Jatene F.B., Bernardo W.M., Stopiglia A.J., Monteiro R, Fantoni D.T., Abduch C.D., Yasbek K.V.B., Irino E.T., Unruh S.M., Kahvegian M. \& Soares A.L.H. 2007. [Viability of the right pneumonectomy in dogs: a parametric, hemogasometric and radiological evaluation.] Viabilidade da pneumonectomia direita em cães: uma avaliação paramétrica, hemogasométrica e radiográfica. Pesquisa Veterinária Brasileira 27(11):447-454. Departamento de Cirurgia, Faculdade de Medicina Veterinária e Zootecnia, Universidade de São Paulo, Av. Prof. Orlando Marques de Paiva 87, São Paulo, SP 05508-900, Brazil. E-mail: edas@uol.com.br

In dogs, the evidence of viability of the right pneumonectomy, as well as the study of complications resulting from surgical procedure, are very important regarding the few specific studies made on the pneumonectomy in this animal species. The objective of this project was to carry out an experimental study to evaluate the viability of the right pneumonectomy in dogs through parametric, hemogasometric and radiographic evaluation. Ten adult healthy mongrel dogs, males and females, weighing $13-32 \mathrm{~kg}$, were used. The dogs were submitted to selective intubation and right thoracotomy at the $5^{\text {th }}$ intercostal space, where the pneumonectomy was performed. A radiografic evaluation was made on days 7,30 and 60 after the surgery, as well as parametric and hemogasometric evaluations were done (before anesthetic induction, 1 hour after extubation, 48 hours and on days 7, 30 and 60 after the surgical procedure), considered important to evaluate possible complications due to the anesthetic and surgical techniques, as well as complications resulting from these procedures. The results were analyzed statistically. Besides the parametric and hemogasometric alterations, all dogs showed compensation for the gaseous exchanges after removing $57 \%$ of the pulmonary volume. Regarding the radiographic evaluation, it could be observed that the expansion of the remaining lung caused heart and lung displacement into the right hemithorax. It can be concluded that the right pneumonectomy is completely feasible in dogs, allowing a parametric, hemogasometric and radiographic satisfactory evaluation in all the animals.
\end{abstract}

INDEX TERMS: Pneumonectomy, lung surgery, dogs.

\footnotetext{
${ }^{1}$ Recebido em 11 de agosto de 2007.

Aceito para publicação em 15 de outubro de 2007.

2 Pós-Graduando, Departamento de Cirurgia, Faculdade de Medicina Veterinária e Zootecnia (FMVZ), Universidade de São Paulo (USP), Av. Prof. Orlando Marques de Paiva 87, São Paulo, SP 05508-900, Brasil. *Autor para correspondência: edas@uol.com.br

${ }^{3}$ Departamento de Cardiopneumologia, Instituto do Coração, Hospital das Clínicas, Faculdade de Medicina (FM), Universidade de São Paulo (USP), Av. Prof. Enéas de Carvalho Aguiar 442, Bloco II, 5ํandar, sala 7, São Paulo, SP 05403-000.

${ }^{4}$ Departamento de Cirurgia, Hospital Veterinário, FMVZ, USP, São Paulo, SP.

${ }^{5}$ Pós-Graduanda, Laboratório de Anatomia Patológica, Instituto do Coração, Hospital das Clínicas, FM, USP, São Paulo, SP.
}

RESUMO.- Em cães, a comprovação da real viabilidade da pneumonectomia direita, bem como, o estudo das complicações resultantes deste procedimento cirúrgico, tornam-se importantes diante da pequena quantidade de estudos na literatura específicos sobre pneumonectomia nesta espécie. 0 objetivo deste trabalho foi realizar um estudo experimental para avaliar a viabilidade da pneumonectomia direita em cães, através da avaliação paramétrica, hemogasométrica e radiográfica Foram utilizados 10 cães adultos, sadios, machos e fêmeas sem raça definida, pesando entre 13 e $32 \mathrm{~kg}$. Todos os cães foram submetidos à intubação seletiva e toracotomia direita no $5^{\circ}$ espaço intercostal, onde foi realizada a pneumonectomia. Foi realizado estudo temporal aos 7, 30 e 60 dias 
de pós-operatório, onde foi feito avaliação radiográfica, bem como, avaliação paramétrica e hemogasométrica (antes da indução anestésica, 1 hora após extubação, 48 horas, 7, 30 e 60 dias após o procedimento cirúrgico), con,siderados importantes para avaliar as possíveis complicações relacionadas com a técnica anestésica, cirúrgica, assim como, as complicações resultantes deste procedimento cirúrgico. Os resultados encontrados foram analisados estatisticamente. Apesar das alterações dos índices paramétricos e hemogasométricos, todos os cães apresentaram compensação das trocas gasosas após retirada de $57 \%$ do volume pulmonar. Na avaliação radiográfica, observamos que a expansão do pulmão remanescente causou deslocamento do coração e pulmão para hemitórax direito. Concluiu-se que a realização da pneumonectomia direita é plenamente viável no cão, permitindo evolução paramétrica, hemogasométrica e radiográfica satisfatória em todos os cães.

TERMOS DE INDEXAÇÃO: Pneumonectomia, cirurgia pulmonar, cães.

\section{INTRODUÇÃO}

As ressecções totais do pulmão podem ser feitas através da pneumonectomia simples, na qual a dissecção dos elementos do hilo pulmonar faz-se sem abrir o pericárdio. A pneumonectomia é indicada nas afeç̧ões que acometem todos os lobos de um pulmão, desde que o pulmão contralateral esteja íntegro. As principais afecções em que a pneumonectomia é indicada são: neoplasias, abscessos e traumas. Dentre as possíveis complicações da pneumonectomia, a hipertrofia ventricular direita, aumento da resistência vascular pulmonar, arritmias, empiema, disfagia e problemas com a cicatrização do coto brônquico são relatados no pós-operatório (Perez et al. 1985, Patel et al. 1992, Fossum 1993, Kopec et al. 1998).

Complicações menos freqüentes, tais como, acidente vascular cerebral, tromboembolismo, síndrome pós-pneumonectomia, fístula esofágica, quilotórax, pneumotórax contralateral, vôlvulo gástrico, pneumopericárdio, paralisia póspneumonectomia, edema pulmonar e falência renal são relatados no pós-operatório, principalmente, após pneumonectomia direita em pacientes humanos (Perez et al. 1985, Patel et al. 1992, Kopec et al. 1998, Bedard et al. 2007).

Em cães a pneumonectomia esquerda e direita corresponde a remover aproximadamente 45 e $57 \%$ do volume pulmonar, respectivamente (Hsia et al. 1994).Segundo Nelson (1993), a pneumonectomia direita em cães resulta em morte na maioria dos casos.

A pneumonectomia pode mimetizar doenças restritivas pulmonares, permitindo o estudo das alterações fisiológicas associadas à perda de grande parte do parênquima pulmonar. A maioria dos estudos devido à perda de tecido pulmonar em cães foram avaliados através de alterações anatômicas medidas no post-mortem e alterações funcionais do pulmão remanescente durante anestesia. Os mecanismos que ocasionam a limitação ao exercício, após ressecção pulmonar, incluem depressão ventilatória, anormalidades nas trocas gasosas devido à desigualdade na relação ventilação/perfusão durante o exercício (Hsia et al. 1990a).
Hsia et al. (1994) e Yan et al. (2005) realizaram cirurgia experimental em cães, estudando as mudanças estruturais no pulmão esquerdo, após pneumonectomia direita em cães. Os resultados dos estudos morfométricos demonstraram uma maior compensação funcional após pneumonectomia direita, quando comparado com a pneumonectomia esquerda.

Em humanos a pneumonectomia direita apresenta maior mortalidade, variando entre 10 e $12 \%$, enquanto nos pacientes submetidos à pneumonectomia esquerda a mortalidade varia entre 1 e 3,5\% (Kopec et al. 1998).A pneumonectomia direita está também associada com maior risco de edema pulmonar com taxa de mortalidade superior a 50\% (Wahi et al. 1989, Kopec et al. 1998).

Hsia et al. (1993), realizaram o primeiro estudo correlacionando compensação morfométrica e fisiológica após exercício em cães. Neste estudo, ocorreu aumento da capacidade de difusão de oxigênio em $27 \%$ após pneumonectomia esquerda quando estimado por técnicas de avaliação fisiológica e morfométrica. As mudanças após pneumonectomia esquerda são hiperinsuflação do pulmão direito, aumento do espaço alveolar e diminuição da barreira alvéolo capilar. Estas mudanças causam um aumento compensatório das trocas gasosas como demonstraram Hsia et al. (1993), Takeda et al. (1996, 1999).

Takeda (1997) demonstrou, através da avaliação tomográfica, que a expansão do pulmão remanescente, após pneumonectomia em cães, pode causar o deslocamento do coração e mediastino para hemitórax esquerdo.

Por meio da pneumonectomia estuda-se o potencial compensatório de vários componentes do pulmão remanescente, incluindo avaliação hemogasométrica e hemodinâmica (Hsia et al. 1990, 1991, 1992; Johnson et al. 1991, Takeda et al. 1996).

Hsia et al. (2001), estudaram os mecanismos compensatórios do pulmão remanescente, após pneumonectomia direita em cães adultos, através da implantação de uma prótese inflável de silicone em hemitórax direito. A expansão pulmonar, volume do tecido septal alveolar, área superficial e capacidade de difusão alvéolo capilar foi significativamente menor nos cães em que a prótese de silicone foi inflada.

Brody et al. (1975) também obtiveram resultados semelhantes ao realizarem pneumonectomia esquerda em camundongos jovens, demonstraram aumento da síntese de DNA nas células alveolares do pulmão remanescente. Uma semana após a cirurgia, houve $38 \%$ de aumento do peso e $41 \%$ de aumento do volume pulmonar.

Pavia et al. (2003) compararam as alterações hemogasométricas e da função estática pulmonar após realização de 3 tipos de ressecção pulmonar (pneumonectomia, lobectomia e ressecção em cunha) em pacientes humanos. Apenas a pneumonectomia esteve associada com a diminuição da resistência aos exercícios, no entanto, as alterações dos parâmetros foram menores que as expectativas dos autores.

Liptak et al. (2004) relataram que a técnica cirúrgica e os resultados da pneumonectomia no pós-operatório não têm sido reportados em cães e gatos. Neste estudo, foi realizado pneumonectomia em 3 cães e 1 gato para tratamento de 
neoplasia pulmonar e doença pleural. Um cão morreu no pósoperatório imediato, provavelmente, devido à insuficiência respiratória. $\mathrm{O}$ gato veio à óbito devido à arritmias cardíacas e progressiva falência cardíaca congestiva. Um cão apresentou complicações gastroentéricas, acompanhado do deslocamento do mediastino e disfunção esofágica. Apenas um cão obteve pós-operatório sem intercorrências.

Riquelme et al. (2005) estudaram as alterações cardiopulmonares, durante anestesia com intubação seletiva, em 7 cães sadios. As avaliações foram realizadas 15 minutos após a realização da intubação seletiva. Durante a mensuração dos parâmetros, houve diminuição significativa da $\mathrm{Pa}_{2}$ e saturação de oxigênio. Não houve alteração significativa dos parâmetros hemodinâmicos e de oxigênio livre. A análise dos resultados demonstrou que o uso da intubação seletiva não causa efeito negativo na oxigenação tecidual.

$O$ presente estudo teve como objetivo avaliar a viabilidade da pneumonectomia direita em cães através da análise paramétrica, hemogasométrica e radiográfica.

\section{MATERIAL E MÉTODOS}

Foram utilizados, neste estudo, 10 cães (Canis familiaris), machos e fêmeas, adultos, sem raça definida, pesando entre 13 e $32 \mathrm{~kg}$, provenientes dos centros de Controle de Zoonoses de cidades pertencentes a grande São Paulo e alojados no Canil do Laboratório de Cirurgia Cardiotorácica da Faculdade de Medicina Veterinária e Zootecnia, Universidade de São Paulo (FMVZ-USP), com 15 dias de antecedência à data da intervenção cirúrgica.

Foram avaliados clinicamente e submetidos a exames complementares através de amostras de sangue coletadas para hemograma, função hepática e renal, para posteriormente serem incluídos no estudo. Esses exames complementares foram novamente realizados com 7 dias de pós-operatório nos 10 cães submetidos à pneumonectomia direita.

Todos os cães foram submetidos à pneumonectomia direita e avaliados com até 60 dias de pós-operatório.

No período que correspondeu a 24 horas antes da cirurgia experimental, foi realizada administração profilática de antibiótico, sendo aplicada benzilpenicilina benzatina na dose de $40.000 \mathrm{U} / \mathrm{kg}$ por via subcutânea.

Após jejum hídrico e alimentar de 6 e 12 horas, respectivamente, os cães receberam como medicação pré-anestésica acepromazina $0,2 \%$ na dose de $0,1 \mathrm{mg} / \mathrm{kg}$, por via intramuscular, 10 minutos antes da indução. A indução anestésica foi realizada através da utilização de propofol na dose de $5 \mathrm{mg} / \mathrm{kg}$ por via intravenosa. Em seguida, foi realizada intubação seletiva, com o auxílio de broncoscópio flexível de 3,2mm de diâmetro (Broncoscópio LF-DPMS-Olympus ${ }^{\circledR}$ ), manutenção anestésica com agente inalatório isofluorano e bloqueio neuromuscular com administração de rocurônio na dose de $0,6 \mathrm{mg} /$ $\mathrm{kg}$ por via intravenosa.

A ventilação mecânica foi regulada de maneira a manter pressão inspiratória média de $20 \mathrm{~mm} \mathrm{Hg}$ na frequiência de 20-25 movimentos por minuto e com fração inspiratória de oxigênio de $100 \%$.

Os cães foram submetidos à toracotomia lateral direita na altura do $5^{\circ}$ espaço intercostal. As costelas foram separadas com afastador tipo Finochietto infantil (Fig.1).

Os pulmões foram afastados lateralmente e dorsalmente em relação à cavidade pleural, sendo identificadas as estruturas do hilo pulmonar.

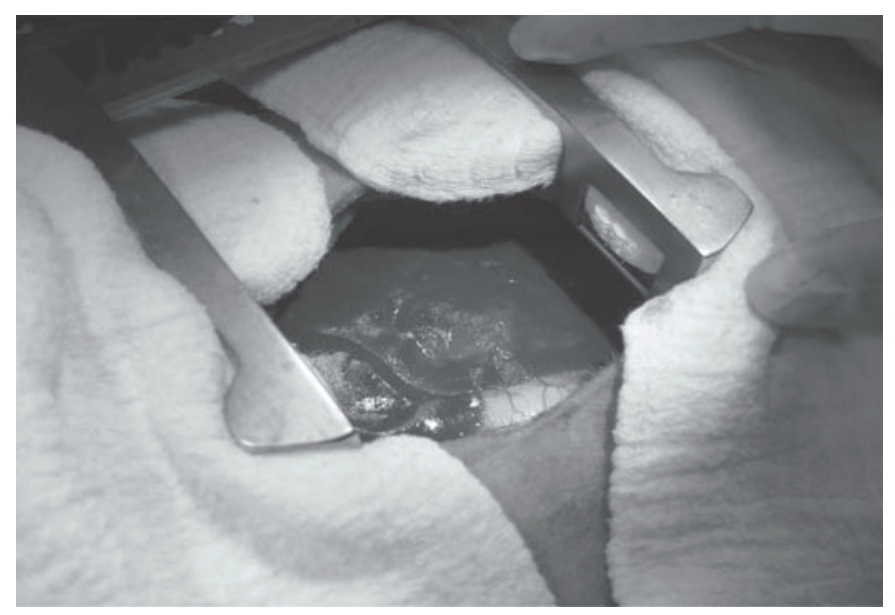

Fig.1. Imagem fotográfica da abordagem cirúrgica através do $5^{\circ}$ espaço intercostal direito. Em detalhe, região de pulmão direito com atelectasia durante intubação seletiva.

Após ligadura dos elementos vasculares do hilo, o brônquio foi dissecado e uma pinça atraumática foi colocada proximalmente ao local da secção. A seguir, o brônquio principal direito foi seccionado e o pulmão ressecado. $O$ coto proximal foi submetido à sutura manual com pontos separados "em 8" utilizando fio polipropileno 5-0.

Após a sutura, a pinça foi retirada, sendo testada a aerostasia através do preenchimento da cavidade pleural com solução fisiológica $0,9 \%$ e, em seguida, foram realizadas manobras de insuflação pulmonar. Não havendo borbulhonamento, a solução salina foi aspirada. Realizada a ressecção pulmonar, foi feita inspeção minuciosa do campo operatório para certificar-se da boa qualidade da hemostasia e aerostasia.Um dreno tubular sob selo d'água foi colocado no espaço pleural.A síntese da parede torácica foi realizada de acordo com técnica convencional. $\mathrm{O}$ dreno torácico foi mantido até que o animal estivesse estável e acordado.

Os cães foram encaminhados ao canil do Laboratório de Cirurgia Cardiotorácica da FMVZ-USP para cuidados pós-operatórios: analgesia, curativos da ferida cirúrgica e anotações dos parâmetros clínicos. Foram mantidos com colar protetor até a retirada dos pontos.

Os cães foram submetidos à avaliação paramétrica clínica e laboratorial através da mensuração da temperatura (TR), hematócrito $(\mathrm{Ht})$, hemoglobina $(\mathrm{Hb})$, freqüência cardíaca $(\mathrm{FC})$ e freqüência respiratória (FR).Da mesma maneira, os cães foram submetidos à avaliação hemogasométrica através da obtenção no sangue arterial dos valores da Pressão Parcial de Oxigênio $\left(\mathrm{Pa}_{2}\right)$, Pressão Parcial de Dióxido de Carbono $\left(\mathrm{PaCO}_{2}\right)$, Concentração Hidrogeniônica $(\mathrm{pH})$, Saturação de Hemoglobina $\left(\mathrm{S}_{\mathrm{a}} \mathrm{O}_{2} \%\right)$ e Bicarbonato Plasmático $\left[\mathrm{HCO}_{3}^{-}\right]$.

Os momentos de mensuração dos parâmetros acima relacionados estão representados no Quadro 1.

Para a avaliação radiográfica os cães foram encaminhados para o Serviço de Diagnóstico por Imagem da FMVZ-USP 24 horas antes e com 7, 30 e 60 dias, após o procedimento cirúrgico, onde foi realizada radiografia torácica ventro-dorsal, látero-lateral direita e esquerda para avaliação dos campos pulmonares, silhueta cardíaca e espaço pleural, visando avaliar as alterações topográficas decorrentes da pneumonectomia e descartar imagens radiográficas de pneumotórax e efusão pleural no pós-operatório.

Avaliação estatística. As variáveis foram inicialmente analisadas descritivamente. Para as variáveis quantitativas (avaliação paramétrica e hemogasométrica), esta análise foi realizada através da observação dos valores mínimos e máximos e do cálculo de médias, 
Quadro 1. Momentos de mensuração das avaliações paramétricas e hemogasométricas nos cães submetidos à pneumonectomia direita

\begin{tabular}{|c|c|c|c|}
\hline Momento Zero & $\left(\mathrm{M}_{0}\right)$ & $\begin{array}{l}\text { Antes do pr } \\
\text { (antes da in }\end{array}$ & $\mathrm{FC}, \mathrm{FR}, \mathrm{TR}, \mathrm{Ht}, \mathrm{Hb}, \mathrm{Pa}_{2}, \mathrm{PaCO}_{2}, \mathrm{pH}, \mathrm{S}_{\mathrm{a}} 0_{2} \% \mathrm{e}\left[\mathrm{HCO}_{3}^{-}\right]$ \\
\hline Mon & $\left(\mathrm{M}_{1}\right)$ & & FC, FR, TR, Ht, $\mathrm{Hb}, \mathrm{Pa}_{2}, \mathrm{PaCO}_{2}, \mathrm{pH}, \mathrm{S}_{\mathrm{a}} 0_{2} \%$ \\
\hline Mome & $\left(\mathrm{M}_{2}\right)$ & 48 horas após & FC, FR, TR, Ht, $\mathrm{Hb}, \mathrm{Pa}_{2}, \mathrm{PaCO}_{2}, \mathrm{pH}, \mathrm{S}_{\mathrm{a}} 0_{2} \%$ \\
\hline Momento Três & $\left(\mathrm{M}_{3}\right)$ & 7 dias após & FC, FR, TR, Ht, $\mathrm{Hb}, \mathrm{Pa}_{2}, \mathrm{PaCO}_{2}, \mathrm{pH}, \mathrm{S}_{\mathrm{a}} 0_{2} \%$ e $\left[\mathrm{HCO}_{3}^{-}\right]$ \\
\hline Momento Quatro & $\left(\mathrm{M}_{4}\right)$ & 30 dias após o procedimento cirúrgico & $\mathrm{FC}, \mathrm{FR}, \mathrm{TR}, \mathrm{Ht}, \mathrm{Hb}, \mathrm{Pa}_{2}, \mathrm{PaCO}_{2}, \mathrm{pH}, \mathrm{S}_{\mathrm{a}} 0_{2} \%$ e $\left[\mathrm{HCO}_{3}^{-}\right]$ \\
\hline Momento Cinco & $\left(\mathrm{M}_{5}\right)^{\mathrm{r}}$ & 60 dias após o procedimento cirúrgico & $\mathrm{FC}, \mathrm{FR}, \mathrm{TR}, \mathrm{Ht}, \mathrm{Hb}, \mathrm{Pa}_{2}, \mathrm{PaCO}_{2}, \mathrm{pH}, \mathrm{S}_{\mathrm{a}} 0_{2} \%$ e $\left[\mathrm{HCO}_{3}^{-}\right]$ \\
\hline
\end{tabular}

desvios-padrão e medianas. Para as variáveis qualitativas (avaliação radiográfica) foram calculadas as frequiências absolutas e relativas.

O comportamento do grupo de cães foi averiguado considerandose as condições estudadas, nos diferentes momentos, em cada mensuração foi utilizado técnica de Análise de Variância com medidas repetidas (Timm 1975), a qual consiste no ajuste de um modelo linear multivariado a partir do qual a seguinte hipótese foi testada: $\mathrm{H}_{\mathrm{O}}$ cujo perfil médio de resposta é paralelo ao eixo das abscissas, ou seja, não há efeito do fator condição de avaliação.

A estatística de Wilks, com a aproximação para a estatística de $\mathrm{F}$, foi utilizada no teste da hipótese acima. A técnica de Análise de Variância com medidas repetidas pressupõe normalidade dos dados, quando esta suposição for rejeitada, as variáveis foram transformadas através do logarítmo.

Caso a normalidade dos dados (mesmo com a variável transformada) não tenha sido observada foi utilizado o teste não paramétrico de Friedman (Rosner 1986).

Todos os testes foram realizados admitindo-se o nível de significância de $5 \%$.

\section{RESULTADOS}

As cirurgias foram realizadas conforme proposto no protocolo cirúrgico.

Comparando-se com os valores controle, foi possível observar diminuição estatísticamente significativa dos valores do hematócrito e hemoglobina nos momentos M1, M2 e M3 (Fig.2 e 3, Quadros 2 e 3).

Em contrapartida, ocorreu aumento estatísticamente significativo, em relação ao momento $\mathrm{M} 0$, nos valores de hematócrito e hemoglobina no momento M5 (Fig.2 e 3, Quadros 2 e 3).

Foi observada diminuição estatísticamente significativa

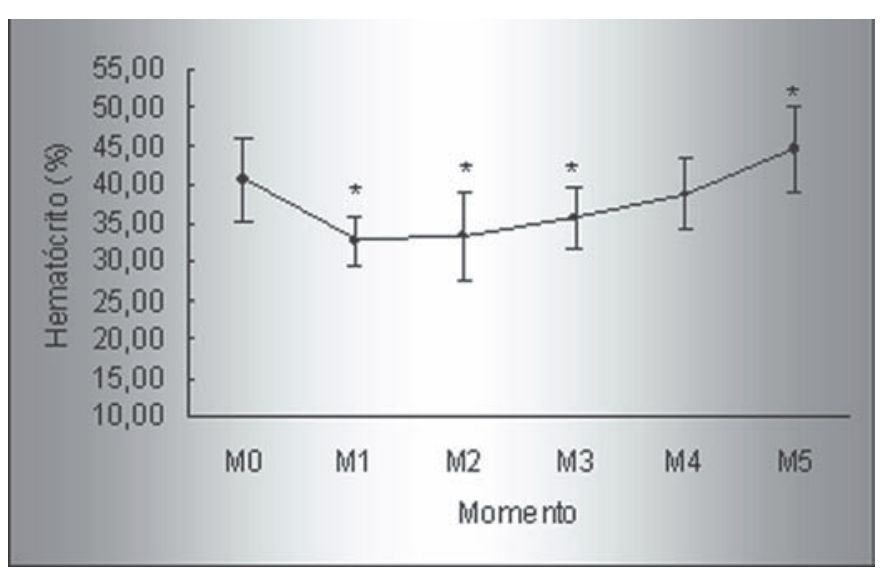

Fig.2. Valores da média e desvio-padrão do hematócrito (\%) dos cães submetidos à pneumonectomia direita. * Diferença significativa do momento M0 $(\mathrm{p}<0,05)$.

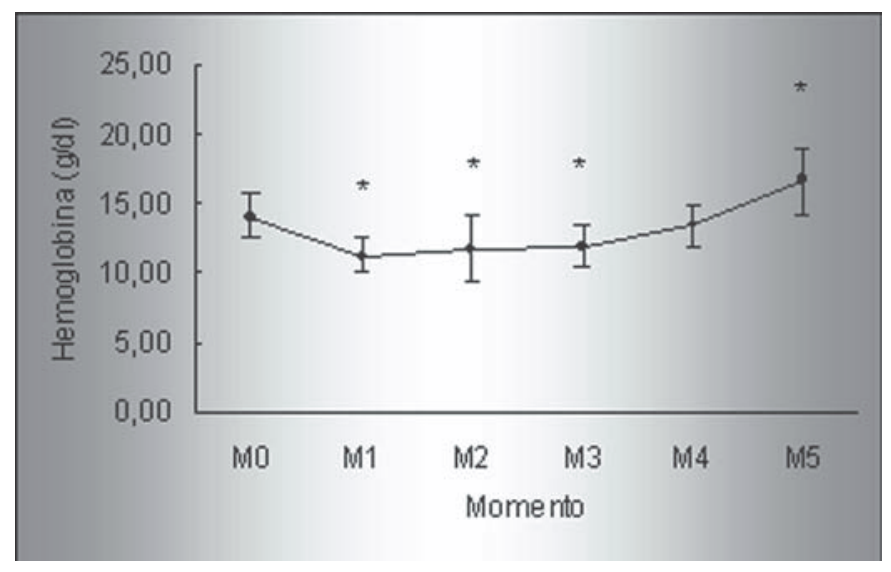

Fig.3. Valores da média e desvio-padrão da hemoglobina (g/dl) dos cães submetidos à pneumonectomia direita. *Diferença significativa do momento M0 $(\mathrm{p}<0,05)$.

dos valores da temperatura, em relação ao M0,no momento M1 (Fig.4, Quadro 4).

Em relação à avaliação hemogasométrica, foi verificada diminuição estatísticamente significativa, apenas nos valores do $\mathrm{pH}$, no momento $\mathrm{M} 1$, com retomada aos valores normais nos demais momentos (Fig.5, Quadro 5).

Quadro 2. Valores da média, desvio-padrão, mediana, mínimo e máximo do hematócrito (\%) dos cães submetidos à pneumonectomia direita

\begin{tabular}{cccccc}
\hline Momento & Média & $\mathrm{dp}$ & Mediana & Mínimo & Máximo \\
\hline M0 & 40,60 & 5,30 & 40,50 & 30,00 & 51,00 \\
M1 ${ }^{*}$ & 32,90 & 3,38 & 34,00 & 27,00 & 38,00 \\
M2 ${ }^{*}$ & 33,40 & 5,66 & 32,50 & 24,00 & 45,00 \\
M3 & 35,80 & 4,02 & 35,50 & 28,00 & 41,00 \\
M4 & 38,90 & 4,58 & 39,50 & 31,00 & 44,00 \\
M5 & 44,70 & 5,46 & 44,50 & 37,00 & 53,00 \\
\hline
\end{tabular}

* Diferença significativa do momento M0 $(p<0,05)$.

Quadro 3. Valores da média, desvio-padrão, mediana, mínimo e máximo da hemoglobina (g/dl) dos cães submetidos à pneumonectomia direita

\begin{tabular}{cccccc}
\hline Momento & Média & $\mathrm{dp}$ & Mediana & Mínimo & Máximo \\
\hline M0 & 14,08 & 1,59 & 14,10 & 10,60 & 16,90 \\
M1 & 11,21 & 1,27 & 11,25 & 9,40 & 13,20 \\
M2 & 11,84 & 2,31 & 11,80 & 8,00 & 17,00 \\
M3 & 12,06 & 1,52 & 12,10 & 9,10 & 14,10 \\
M4 & 13,43 & 1,47 & 14,05 & 10,70 & 14,90 \\
M5 * & 16,69 & 2,43 & 17,25 & 12,60 & 19,80 \\
\hline
\end{tabular}

* Diferença significativa do momento M0 $(p<0,05)$. 


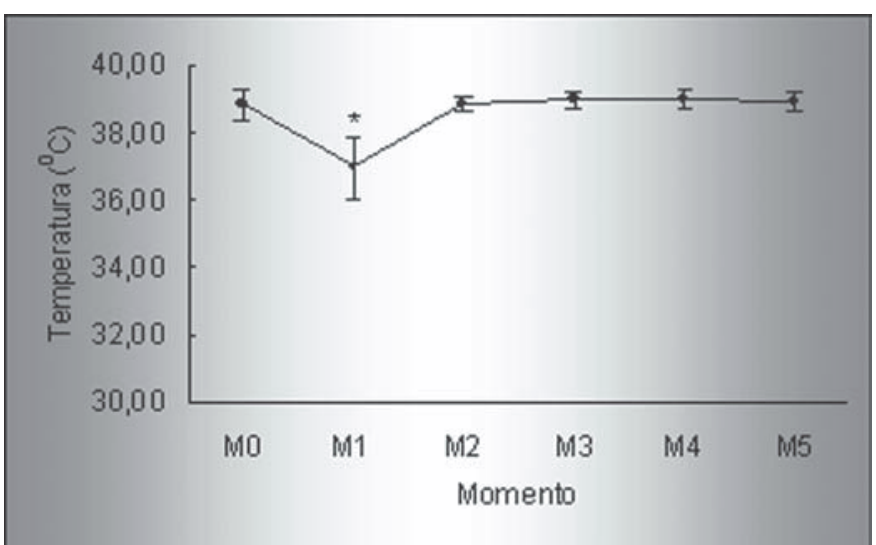

Fig.4. Valores da média e desvio-padrão da temperatura $\left({ }^{0} \mathrm{C}\right)$ dos cães submetidos à pneumonectomia direita. "Diferença significativa do momento M0 $(\mathrm{p}<0,05)$.

Quadro 4. Valores da média, desvio-padrão, mediana, mínimo e máximo da temperatura $\left({ }^{\circ} \mathrm{C}\right)$ dos cães submetidos à pneumonectomia direita

\begin{tabular}{cccccc}
\hline Momento & Média & $\mathrm{dp}$ & Mediana & Mínimo & Máximo \\
\hline M0 & 38,83 & 0,44 & 38,95 & 38,00 & 39,30 \\
M1 & 36,94 & 0,93 & 37,05 & 35,30 & 38,00 \\
M2 & 38,84 & 0,25 & 38,80 & 38,50 & 39,30 \\
M3 & 38,96 & 0,25 & 39,00 & 38,50 & 39,20 \\
M4 & 39,00 & 0,27 & 39,00 & 38,50 & 39,30 \\
M5 & 38,90 & 0,30 & 39,00 & 38,50 & 39,30
\end{tabular}

*Diferença significativa do momento M0 $(p<0,05)$.

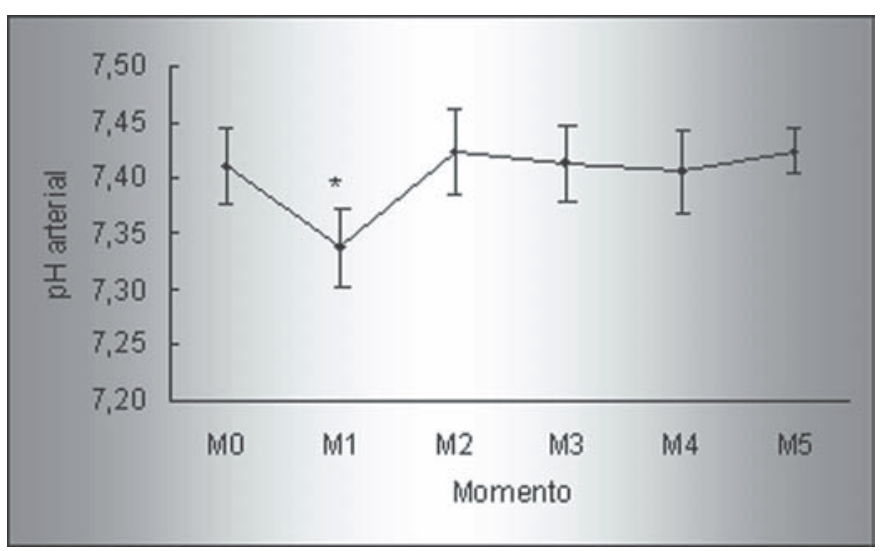

Fig.5. Valores da média e desvio-padrão do $\mathrm{pH}$ arterial dos cães submetidos à pneumonectomia direita. *Diferença significativa do momento M0 $(\mathrm{p}<0,05)$.

Quadro 5. Valores da média, desvio-padrão, mediana, mínimo e máximo do $\mathrm{pH}$ arterial dos cães submetidos à pneumonectomia direita

\begin{tabular}{cccccc}
\hline Momento & Média & $\mathrm{dp}$ & Mediana & Mínimo & Máximo \\
\hline M0 & 7,41 & 0,03 & 7,40 & 7,36 & 7,46 \\
M1 & 7,34 & 0,04 & 7,33 & 7,29 & 7,42 \\
M2 & 7,42 & 0,04 & 7,41 & 7,39 & 7,49 \\
M3 & 7,41 & 0,03 & 7,41 & 7,37 & 7,47 \\
M4 & 7,41 & 0,04 & 7,41 & 7,37 & 7,47 \\
M5 & 7,42 & 0,02 & 7,42 & 7,40 & 7,46
\end{tabular}

* Diferença significativa do momento M0 $(p<0,05)$.

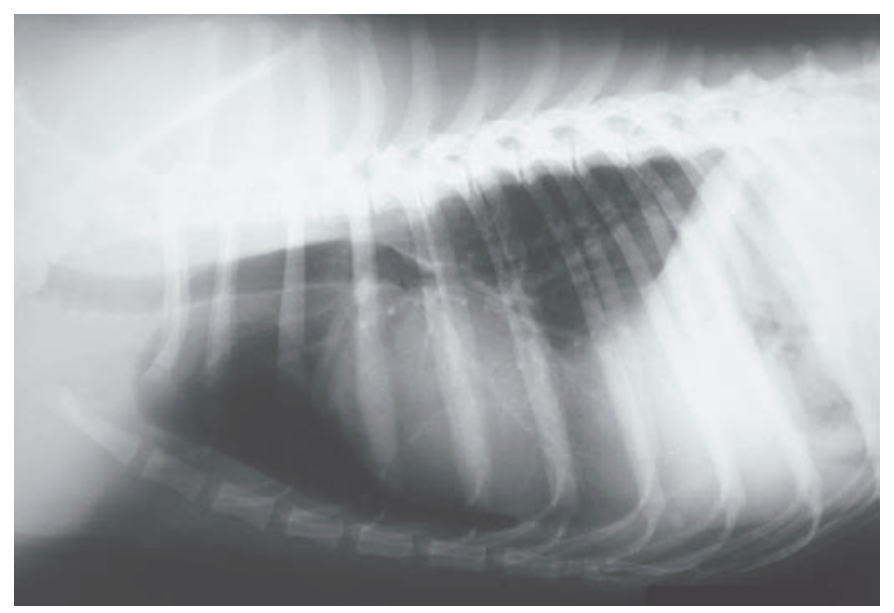

Fig.6. Imagem radiográfica em projeção látero-lateral direita de cão após pneumonectomia direita. Observa-se deslocamento dorsal da silhueta cardíaca devido à hiperinsuflação de campos pulmonares esquerdos.

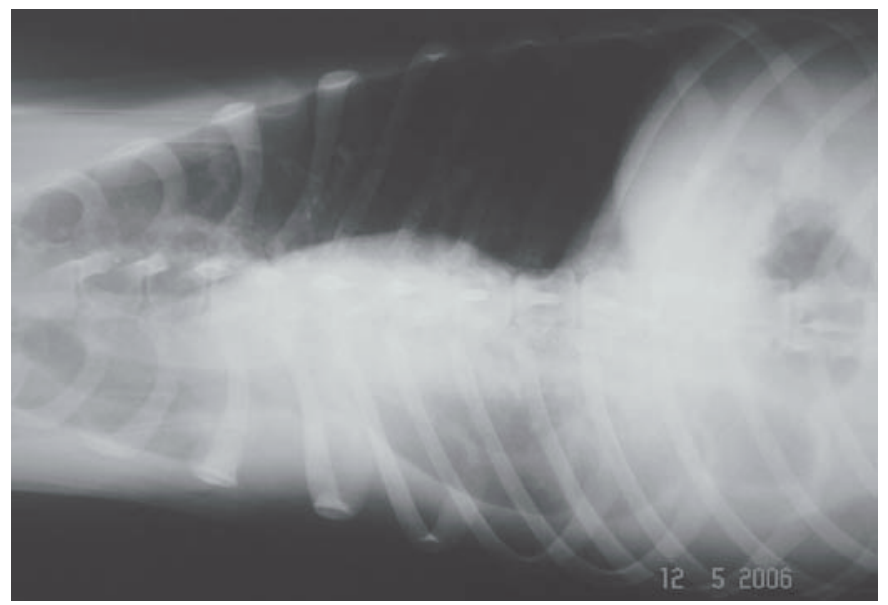

Fig.7. Imagem radiográfica em projeção ventro dorsal de cão após pneumonectomia direita. Observa-se hiperinsuflação de campos pulmonares esquerdos, invadindo o hemitórax direito cranial e caudalmente e desvio lateral direito da traquéia e silhueta cardíaca.

As possíveis alterações topográficas das estruturas presentes na cavidade torácica, tais como, deslocamento da silhueta cardíaca, mediastino e hiperinsuflação de lobos pulmorares esquerdos foram avaliados radiograficamente (Fig.6 e 7).

Nos 10 cães estudados, nenhum apresentou alterações radiográficas de tórax no pré-operatório.

As radiografias de tórax na projeção ventro-dorsal apresentaram imagens de hiperinsuflação de campos pulmonares esquerdos, em sete cães aos 7 dias de pós-operatório e nove cães aos 30 e 60 dias de pós-operatório.

Em cinco cães, aos 7 dias de pós-operatório, e seis cães, aos 30 e 60 dias de pós-operatório, as radiografias de tórax na projeção látero-lateral apresentaram desvio dorsal da silhueta cardíaca, sugerindo hiperinsuflação de campos pulmonares esquerdos.

Os Cães 3, 4, 6, 7 e 9 apresentaram efusão pleural aos 7 dias de pós-operatório. 
Em $80 \%$ dos cães aos 7 dias de pós-operatório e em $100 \%$ dos cães aos 30 e 60 dias de pós-operatório, as radiografias de tórax na projeção ventro-dorsal demonstraram deslocamento da silhueta cardíaca e mediastino para hemitórax direito.

\section{DISCUSSÃO}

O estudo da viabilidade da pneumonectomia direita em cães, através da avaliação paramétrica, hemogasométrica e radiográfica, está justificado diante do interesse crescente e da escassez de trabalhos em Medicina Veterinária, considerando os aspectos referentes à padronização da técnica anestésica, cirúrgica, bem como, as complicações resultantes deste procedimento cirúrgico.

Concomitantemente, deve-se considerar a alta mortalidade após a pneumonectomia direita em pacientes humanos, variando entre 10 e $13 \%$ quando comparada com a pneumonectomia esquerda, onde temos mortalidade entre 1 e 3,5\% (Kopec et al. 1998, Muysons et al. 1998).

Na rotina da cirurgia em Medicina Veterinária, existem casos nos quais não há uma conduta bem estabelecida, muitas vezes o tratamento clínico seguido de lobectomia é insuficiente, limitado e paliativo.

Assim, nas intervenções cirúrgicas do pulmão, sejam por trauma, infecção ou neoplasia, a pneumonectomia pode ser um importante aliado no protocolo cirúrgico.

No presente estudo, todos os cães completaram os procedimentos metodológicos. Estes resultados não coadunaram com o que fora relatado por Nelson (1993), onde afirma que a pneumonectomia direita em cães é quase sempre fatal.

Nos 10 cães operados, o acesso escolhido através da incisão da pele e tecido subcutâneo na altura do $5^{\circ}$ espaço intercostal, proporcionou um campo operatório adequado para a abordagem dos elementos do hilo pulmonar. No entanto, apesar da intubação seletiva, o volume pulmonar maior do lado direito, que corresponde aproximadamente a 57\% do volume pulmonar total, dificultou a dissecção e ligadura das veias pulmonares. Além disso, a localização anatômica da artéria pulmonar direita dificultou sua exposição cirúrgica. A artéria pulmonar direita transita ventralmente à carina, emergindo no hilo situado entre os lobos pulmonares cranial e médio direitos, acompanhando dorsalmente o brônquio do lobo pulmonar médio, sendo observada dorsalmente às veias pulmonares (Hare,1986;Nelson, 1993).Entretanto, de acordo com Riquelme et al. (2005), no presente estudo, a intubação seletiva facilitou sobremaneira as manobras na cavidade torácica e exposição dos elementos do hilo pulmonar durante o procedimento cirúrgico.

Em relação a técnica anestésica, foi escolhido o isofluorano por permitir rápida indução e recuperação anestésica, apresentando pequena taxa de metabolização, sendo mínimos os seus efeitos cardiovasculares (Oliva 2002). As características do anestésico utilizado permitiram rápida recuperação anestésica com retomada da respiração espontânea logo após o término do procedimento cirúrgico. Verificou-se estabilidade cardiovascular ao longo do procedimento,demonstrando adequação da técnica anestésica empregada.
A utilização de um broncofibroscópio flexível de 3,2mm de diâmetro facilitou a realização da intubação seletiva permitindo, devido ao seu diâmetro reduzido, uma ventilação adequada, minimizando a queda da saturação do oxigênio durante o procedimento, de acordo com Sackner (1975).

No que se refere aos valores do hematócrito e hemoglobina, observamos queda significativa desses valores nos momentos M1, M2 e M3 (uma hora após a extubação, 48 horas e 7 dias após o procedimento cirúrgico) em relação aos valores basais. Acredita-se que a ocorrência de hemodiluição devido à administração de fluidoterapia durante o procedimento cirúrgico, poderia explicar a queda desses valores.

Todavia, verificou-se incremento significativo nos valores do hematócrito e hemoglobina no momento M5 (60 dias após o procedimento cirúrgico). De fato, Hsia et al. (1994) e Hsia et al. (1990) constataram policitemia após realização de pneumonectomia experimental em cães, afirmando ser um importante mecanismo compensatório para tentar manter as mesmas taxas de trocas gasosas no período pré-operatório.

O procedimento anestésico ocasionou queda estatisticamente significativa da temperatura no momento M1 (uma hora após extubação) com retorno aos valores normais no pósoperatório. A diminuição da temperatura no momento M1 ocorreu devido à diminuição do metabolismo basal durante a anestesia.

A despeito das intercorrências intra e pós-operatórias, associadas a alta taxa de mortalidade,citadas por Liptak et al.(2004) e Nelson (1993),após pneumonectomia direita em cães,o presente estudo mostrou uma rápida e efetiva compensação funcional do pulmão esquerdo,ou seja,este foi capaz de compensar funcionalmente o pulmão retirado através da hiperinsuflação pulmonar de acordo com (Hsia et al. 1990a,b, 1993, 1994a,b, 2001, Johnson et al. 1991, Takeda et al. 1996, 1999, Yan et al. 2005).

Neste estudo,a gasometria comprovou os mecanismos compensatórios após a realização da pneumonectomia direita. Decorridos 48 horas e 7 dias após o procedimento cirúrgico, obtidos com $\mathrm{F}_{\mathrm{i}} \mathrm{O}_{2}$ de $21 \%$, a média da $\mathrm{P}_{\mathrm{a}} \mathrm{O}_{2}$ foi igual a $84,4 \mathrm{mmHg}$ e $83,10 \mathrm{mmHg}$, respectivamente, o que resulta em uma relação de $\mathrm{P}_{\mathrm{a}} \mathrm{O}_{2} / \mathrm{F}_{\mathrm{i}} \mathrm{O}_{2}$ de 401,9 e 396. Estes valores estão dentro da normalidade,tendo-se em vista que a oxigenação é adequada quando a relação $\mathrm{P}_{\mathrm{a}} \mathrm{O}_{2} / \mathrm{F}_{\mathrm{i}} \mathrm{O}_{2}$ é superior a 280 (Rossi et al. 1998), demonstrando uma rápida compensação do pulmão esquerdo, mantendo os valores de $\mathrm{P}_{\mathrm{a}} \mathrm{O}_{2}$ e relação $\mathrm{P}_{\mathrm{a}} \mathrm{O}_{2} /$ $\mathrm{F}_{\mathrm{i}} \mathrm{O}_{2}$ normais até o final do pós-operatório.

No presente trabalho, a realização da pneumonectomia direita ocasionou acidose respiratória transitória, demonstrada pela redução significativa do pH no momento M1 (uma hora após a extubação). Pode-se pressupor que neste momento, as alterações nos valores do $\mathrm{pH}$ foram causadas durante mecanismo de adaptação da ventilação alveolar e sua adequação para manter esses valores em níveis normais, após a retirada abrupta do pulmão direito. Estes resultados vão de encontro aos trabalhos realizados por Hsia et al. (1994), Takeda et al. $(1996,1999)$ no pós-operatório imediato após pneumonectomia esquerda em cães.

O estudo radiográfico do tórax foi um procedimento im- 
portante para se avaliar as alterações anatômicas após a pneumonectomia direita e possíveis alterações do parênquima pulmonar, presença de pneumotórax e efusão pleural no pósoperatório.

No estudo em tela, apenas 5 cães apresentaram efusão pleural na avaliação radiográfica aos 7 dias de pós-operatório. Em contrapartida, Adams et al. (1945), realizaram estudo radiográfico após pneumonectomia em pacientes humanos e observaram aumento gradativo da efusão pleural no pós-operatório. Afirmaram que o fluído pleural se acumula na taxa de 2 espaços intercostais por dia. Todavia, a velocidade de formação de fluído pleural pode variar. Normalmente, $80 \%$ a $90 \%$ do espaço pleural é preenchido em 2 semanas. Analogamente, Christiansen et al. (1965), relataram que o tempo médio para a completa opacificação foi de 3,9 meses, após a realização de pneumonectomia em pacientes humanos.

No presente estudo, acredita-se que a causa de efusão pleural no pós-operatório foi o resíduo de solução salina $0,9 \%$ durante o teste de aerostasia da sutura do brônquio principal direito associado com processo inflamatório exsudativo nos primeiros dias de pós-operatório.

Os exames radiográficos na fase pós-operatória demonstraram imagens de desvio dorsal da silhueta cardíaca e deslocamento do coração e mediastino para hemitórax direito, sugerindo hiperinsuflação de campos pulmonares esquerdos.

Considerando os resultados obtidos, concordamos com Takeda et al. (1997) quando demonstraram através da avaliação tomográfica que a expansão do pulmão remanescente, após pneumonectomia esquerda em cães, pode causar o deslocamento do coração e pulmão para hemitórax esquerdo.

Estes resultados vêm de encontro com os trabalhos conduzidos por Hsia et al. (1993), Takeda et al. $(1996,1999)$ onde verificaram que as mudanças após pneumonectomia esquerda em cães são hiperinsuflação do pulmão direito, aumento do espaço alveolar e diminuição da barreira alvéolo capilar, causando um aumento compensatório das trocas gasosas.

Desta forma, esperamos que este trabalho estimule a utilização da pneumonectomia direita na rotina dos centros cirúrgicos veterinários.

A comprovação que o pulmão remanescente consegue manter a capacidade de oxigenação adequada do sangue, permite que este princípio seja utilizado na rotina cirúrgica em pequenos animais, podendo ser uma alternativa viável e tecnicamente segura, elevando a probabilidade de melhora da qualidade de vida e cura desses animais, além de se constituir um interessante instrumento para estudos experimentais mais aprofundados da fisiologia pulmonar.

\section{CONCLUSÕES}

A avaliação dos resultados obtidos permitiu concluir que a técnica cirúrgica utilizada para a realização da pneumonectomia direita é plenamente viável no cão. Este modelo experimental pode ser realizado através de técnica cirúrgica reprodutível nos cães acometidos por doenças pulmonares.

Não foram observadas alterações importantes na hemogasometria em todos os cães operados, demonstrando uma hematose adequada do pulmão remanescente no pós-operatório imediato e tardio.

Os volumes pulmonares foram mantidos no período pósoperatório pelo pulmão remanescente.

Os dados obtidos da avaliação radiográfica permitiram concluir que a pneumonectomia direita promove alterações significativas na anatomia topográfica das estruturas presentes na cavidade torácica, demonstradas por imagens de hiperinsuflação do pulmão esquerdo, deslocamento da silhueta cardíaca e mediastino para hemitórax direito.

Agradecimentos.- Os autores agradecem o apoio financeiro do CNPq e da FAPESP. Trabalho aprovado pela Comissão de Bioética (Protocolo 475/2004).

\section{REFERÊNCIAS}

Bedard E.L., UY K. \& Keshavjee S. 2007. Postpneumonectomy syndrome: a spectrum of clinical presentations. Animals of Thoracic Surgery 83(3):11851186.

Fossum T.W. 1993. Respiratory system, p.2231-2244. In: Slatter D. (ed.), Textbook of Small Animal Surgery. W.B. Saunders, Philadelphia.

Hare W.L.D. 1986. Sistema Respiratório, p.1465-1480. In:_(ed.), Anatomia dos Animais Domésticos, Guanabara Koogan, Rio de Janeiro.

Hsia C.C.W., Carlim J.I., Cassidy S.S., Ramanathan M. \& Johnson R.L. 1990. Hemodynamic changes after pneumonectomy in the exercising foxhound. J. Appl. Physiol. 69(1):51-67.

Hsia C.C.W., Carlim J.I., Wagner P.D., Cassidy S.S. \& Johnson R.L. 1990a. Gas exchange abnormalities after pneumonectomy in conditioned, foxhounds. J. Appl. Physiol. 68(1):94-104.

Hsia C.C.W., Doffey F.F., Stalder-Navarro V., Johnson R.L., Reynolds R.C. \& Weibel E.R. 1993. Structural changes underlying compensatory increase of diffusing capacity after left pneumonectomy in adult dogs. J. Clin. Invest. 92:758-764.

Hsia C.C.W., Herazo L.F., Doffey F.F. \& Weibell E.R. 1994. Compensatory lung growth occurs in adult dogs after right pneumonectomy. J. Clin. Invest. 94(1):405-412.

Hsia C.C.W., Herazo L.F., Ramanathan M. \& Johnson R.L. 1994. Cardiopulmonary adaptations to pneumonectomy in dogs. IV. Membrane diffusing capacity and capillary blood volume. J. Appl. Physiol. 77(2):998-1005.

Hsia C.C.W., Ramanathan M., Pean J.L. \& Johnson R.L. 1992. Respiratory muscle blood flow in exercising dogs after pneumonectomy. J. Appl. Physiol. 73(1):240-247.

Hsia C.C.W., Wu E.Y., Wagner E. \& Weibel E.R. 2001. Preventing mediastinal shift after pneumonectomy impairs regenerative alveolar tissue growth. Am. J. Physiol. Lung Cell Mol. Physiol. 281(5):279-287.

Johnson R.L., Cassidy S.S., Grover R., Ramanathan M., Estrera A., Reynolds R.C., Epstein R. \& Schutte J. 1991. Effect of pneumonectomy on the remaining lung in dogs. J. Appl. Physiol. 70(2):849-858.

Kopec S.E., Irwin R.S., Umali-Torres C.B., Balikian J.P. \& Conlan A.A. 1998. The postpneumonectomy state. Chest 114:1158-1184.

Liptak J.M., Monnet E., Dernell W.S., Rizzo S.A. \& Withrow S.J. 2004. Pneumonectomy: four cases studies and a comparative review. J. Small Anim. Pract. 45(9):441-447.

Muysoms F.E., Rivière A.B., Defauw J.J., Dossche K.M., Knaepen P.J., Swieten H.A. \& Bosch J.M.M. 1998. Completion pneumonectomy: analysis of operative mortality and survival. Annals Thorac. Surg. 66(4):1165-1169.

Nelson A.W. 1993a. Respiratory system, p.692-708. In: Slatter D. (ed.), Textbook of Small Animal Surgery. W.B. Saunders, Philadelphia.

Nelson A.W. 1993b. Respiratory system, p.777-804. In: Slatter D. (ed.), Textbook of Small Animal Surgery. W.B. Saunders, Philadelphia.

Oliva V.N.L.S. 2002. Anestesia inalatória, p.174-183. In: Fantoni D.T. \& Cortopassi S.R.G. (ed.), Anestesia em Cães e Gatos. Roca, São Paulo.

Patel R.L., Townsend E.R. \& Fountain S.W. 1992. Elective pneumonectomy: 
factors associated with morbidity and operative mortality. Annals Thorac. Surg. 54:84-88.

Pavia R., Monaco F., Mondello B., Barresi P., Barone M., Familiari D. \& Monaco M. 2003. Evaluation of lung function as a prognostic index before and after surgical ressection due to NSCLC. Minerva Chir. 58(4):551-555.

Perez G.F. \& Holland J.N. 1985. Epidural hematoma as a cause of post pneumonectomy paraplegia. Annals Thorac. Surg. 39:282.

Riquelme M., Monnet E., Kudnig S.T., Gaynor J.S., Wagner A.E., Corliss D. \& Salman D.M. 2005. Cardiopulmonary changes induced during one lung ventilation in anesthetized dogs with a closed thoracic cavith. Am. J. Vet. Res. 66(6):973-977.

Rosner B. 1986. Fundamentals of Biostatistics. 2nd ed. PWS Publishers, Boston. 588p.

Rossi N., Kolobow T., Aprigliano M., Tsuno K. \& Giacomini M. 1998. Intratracheal pulmonary ventilation at low airway pressures in a ventilatorinduced model of acute respiratory failure improves lung function and survival. Chest 114:1147-1157.
Sackner M.A. 1975. Bronchofiberscopy: state of the art. Am. Rev. Resp. Dis. 111(1):62-88.

Takeda S., Ramanathan M., Estrera A.S. \& Hsia C.C.W. 1999. Postpneumonectomy alveolar growth does not normalize hemodynamic and mechanical function. J. Appl. Physiol. 87(2):491-497.

Takeda S., Wu E.Y., Epstein R.H., Estrera A.S. \& Hsia C.C.W. 1997. In vivo assessment of changes in air and tissue volumes after pneumonectomy. J. Appl. Physiol. 82(4):1340-1348.

Takeda S., Wu E.Y., Ramanathan M., Estrera A.S. \& Hsia C.C.W. 1996. Temporal course of gas exchange and mechanical compensation after right pneumonectomy in immature dogs. J. Appl. Physiol. 80(4):1304-1312.

Wahi R.M. \& Decaro L.F. 1989. Determinants of perioperative morbidity and mortality after pneumonectomy. Annals Thorac. Surg. 48:33-37.

Yan X., Bellotto D.J., Dane D.M., Elmore R.G., Johnson R.L., Estrera A.S. \& Hsia C.C. 2005. Lack of response to all-transacid supplementation in adult dogs following left pneumonectomy. J. Appl. Physiol. 99(5):16811688. 